\title{
Influence of stoichiometry and aging at operating temperature on thermoelectric Higher Manganese Silicides
}

\author{
Sylvain Le Tonquesse,${ }^{\dagger}$ Loic Joanny, ${ }^{\ddagger}$ Quansheng Guo, ${ }^{\dagger}$ Erik Elkaim, ${ }^{\mathbb{T}}$ \\ Valérie Demange,,$^{\ddagger}$ David Berthebaud, ${ }^{\dagger}$ Takao Mori, ${ }^{\S}$ Mathieu Pasturel, ${ }^{*,+}$ \\ and Carmelo Prestipino*,\$ \\ $\dagger C N R S$ - Saint-Gobain - NIMS, UMI3629, Laboratory for Innovative Key Materials and \\ Structures (LINK), National Institute for Materials Science, 1-1 Namiki, Tsukuba, Ibaraki \\ 305-0044, Japan \\ †Univ Rennes, CNRS, ISCR-UMR6226/ScanMAT-UMS2001, F-35000, Rennes, France \\ IISynchrotron SOLEIL, L'Orme des Merisiers, BP 48, St Aubin, 91192 Gif sur Yvette, France \\ §National Institute for Materials Science (NIMS), WPI-MANA and CFSN, Tsukuba, Japan \\ E-mail: mathieu.pasturel@univ-rennes1.fr; carmelo.prestipino@univ-rennes1.fr
}

\begin{abstract}
Thermoelectric Higher Manganese Silicides, $\mathrm{MnSi}_{x}$, were synthesized by magnesioreduction followed by spark plasma sintering with different nominal compositions $(x=1.65,1.74,1.80)$ and various post-synthesis annealing durations $(0 \mathrm{~h}, 48 \mathrm{~h}, 96 \mathrm{~h}$ and $336 \mathrm{~h}$ ). The composite Nowotny Chimney-Ladder crystal structures of the resulting samples were investigated by synchrotron X-ray powder diffraction. The modulation vector component $\gamma$, generally considered corresponding to the stoichiometry $(x)$ of
\end{abstract}


the material, were accurately determined by Rietveld refinement using a $(3+1) \mathrm{D}$ superspace approach. Regardless of the initial nominal composition, all the samples have a similar $\gamma \sim 1.736$ after $48 \mathrm{~h}$ annealing at $900 \mathrm{~K}$. This result suggests that $\mathrm{MnSi}_{x}$, at the temperature of $900 \mathrm{~K}$, is better described as a defined compound with $\mathrm{x}$ close to 1.736, rather than intermediate solid-solution phases with $1.725<x<1.75$ as predicted by the commonly accepted phase diagram. At the fixed nominal composition $\mathrm{MnSi}_{1.74}, \gamma$ increases significantly from $1.7313(2)$ to $1.7411(1)$ after $336 \mathrm{~h}$ annealing, indicating that the thermal history influences the Si stoichiometry. The evolution of $\gamma$ with time is concomitant with a power factor drop ( $-19 \%)$, attributed to a decrease of the charge carrier concentration. The drop of the power factor, partially compensated by a decrease of the thermal conductivity, results in a $-12 \%$ reduction of the maximum figure of merit $Z T$, after prolonged annealing under realistic application conditions.

\section{Keywords}

HMS, Thermoelectric, Higher Manganese Silicides, Incommensurate crystallography, Microstructure 

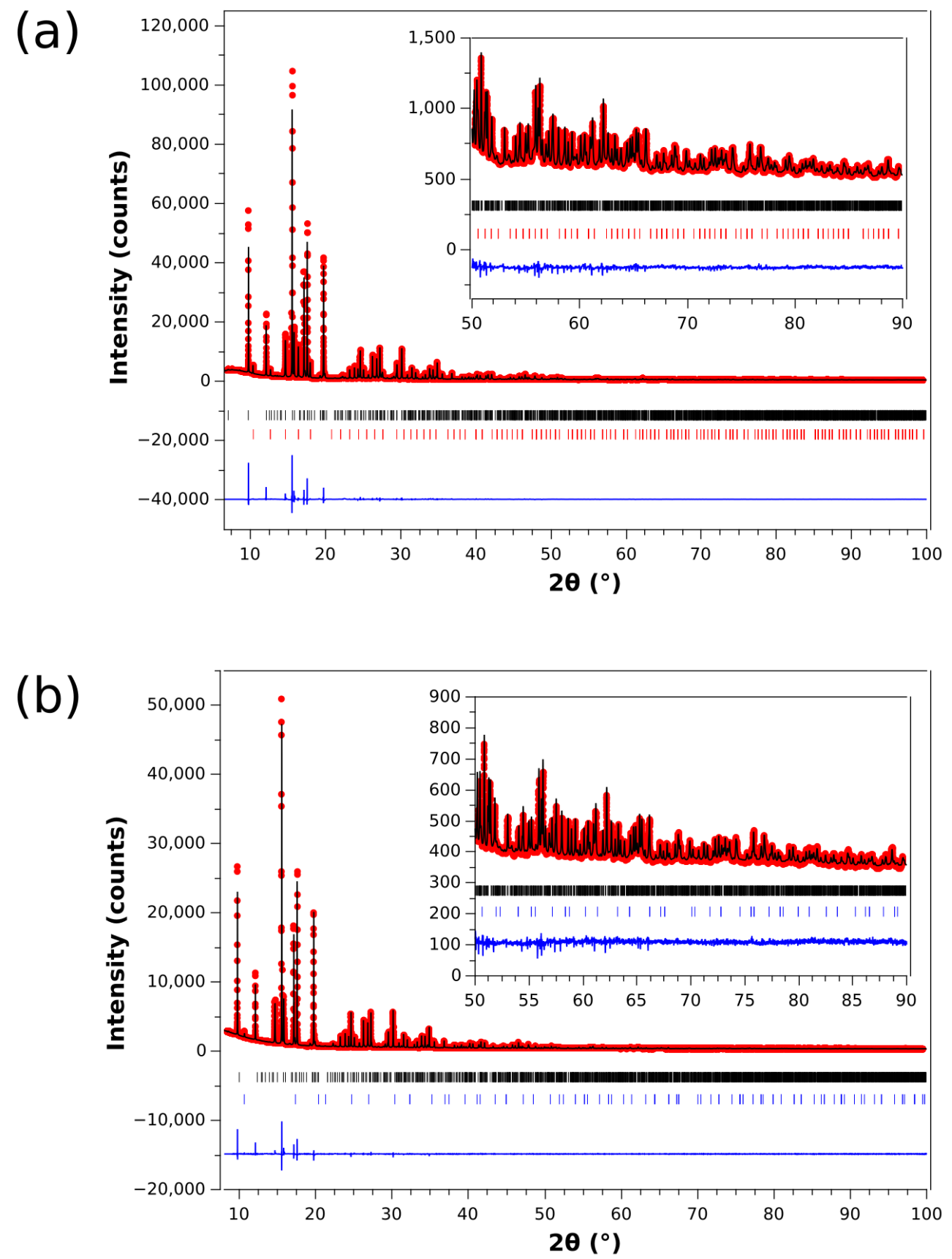

Figure SI. 1: Rietveld refined synchrotron powder XRD patterns of (a) $\mathrm{MnSi}_{1.65}-48 \mathrm{~h}$ and (b) $\mathrm{MnSi}_{1.80}-48 \mathrm{~h}(\lambda=0.58143 \AA)$. The experimental data are plotted in red symbols, the calculated pattern with a black line and the difference with a blue line. The vertical ticks indicate the theoretical Bragg positions of HMS (black), MnSi (red) and Si (blue). The inset is a magnified view of the high angular region of the refined pattern. 


\section{Si $K \alpha_{1}$}

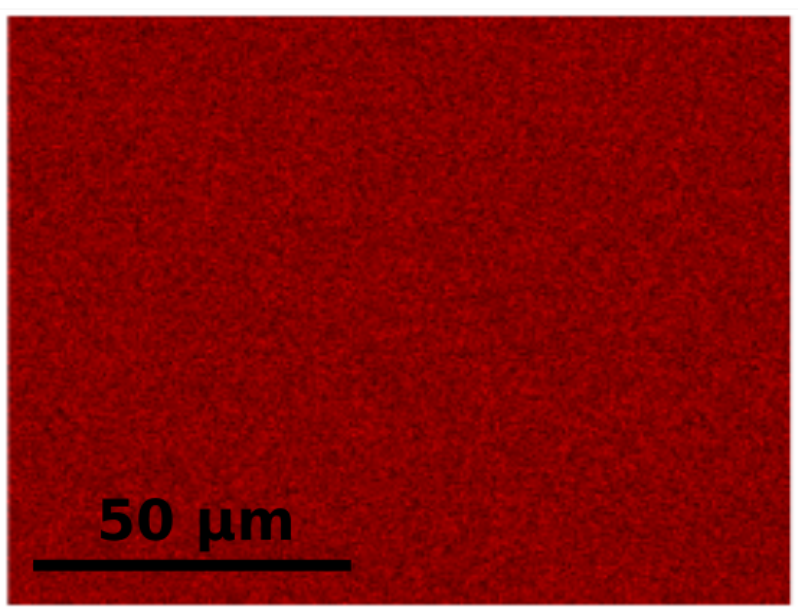

$M n K \alpha_{1}$

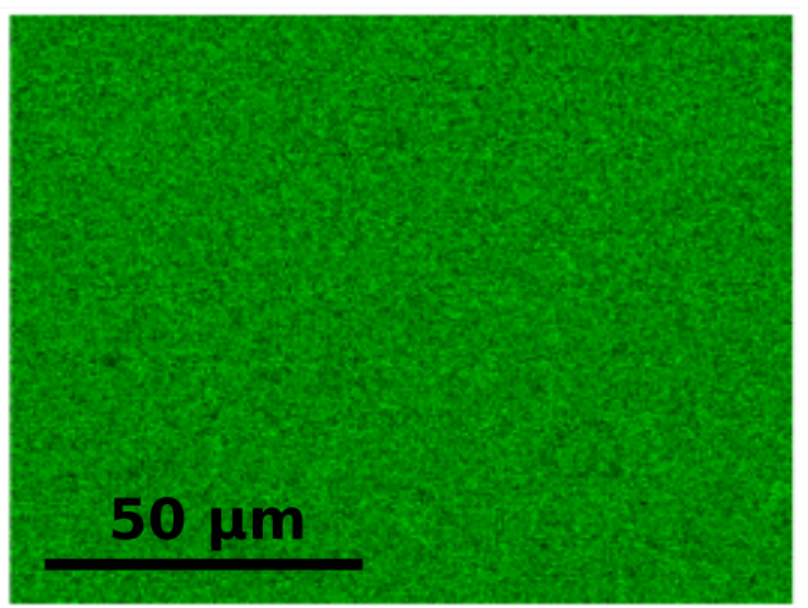

Figure SI. 2: Si and Mn SEM-EDS elemental maps of $\mathrm{MnSi}_{1.74}-48 \mathrm{~h}$ 
(a)

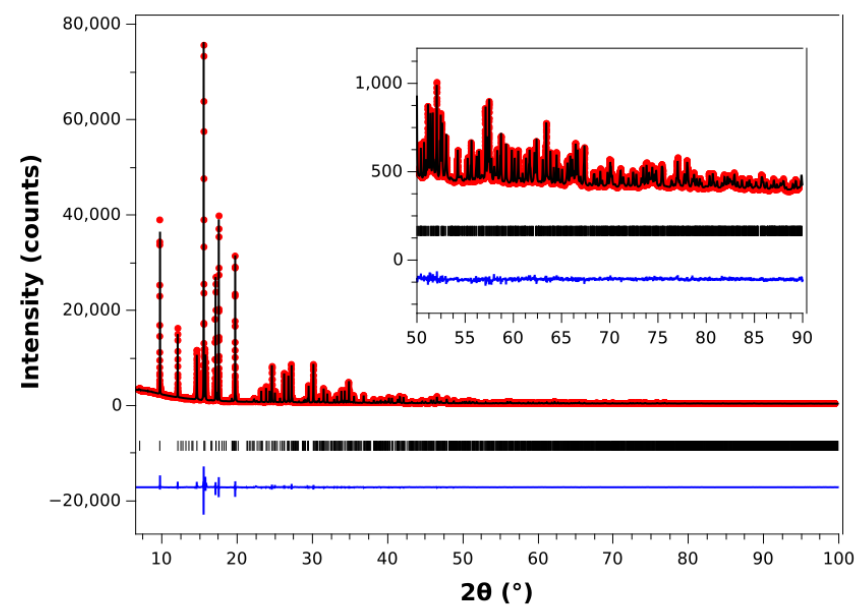

(b)

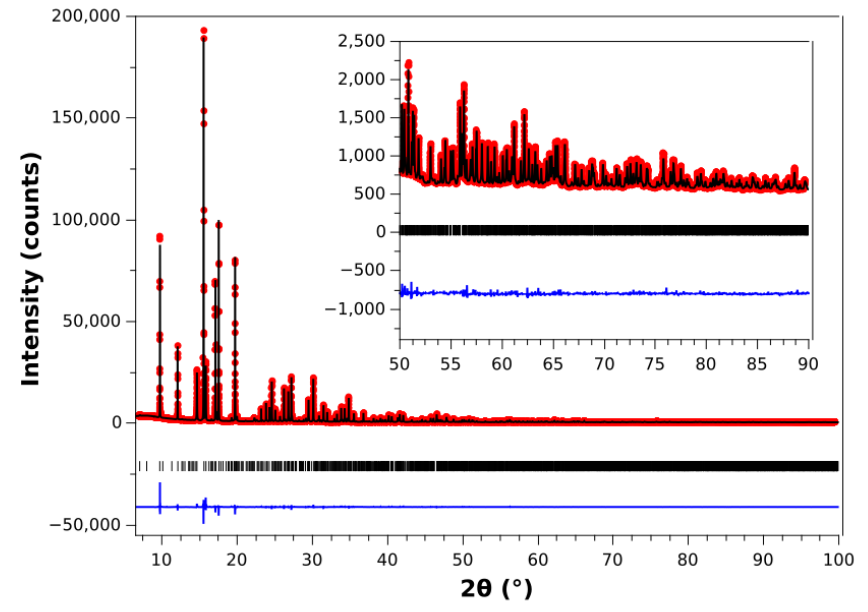

(c)

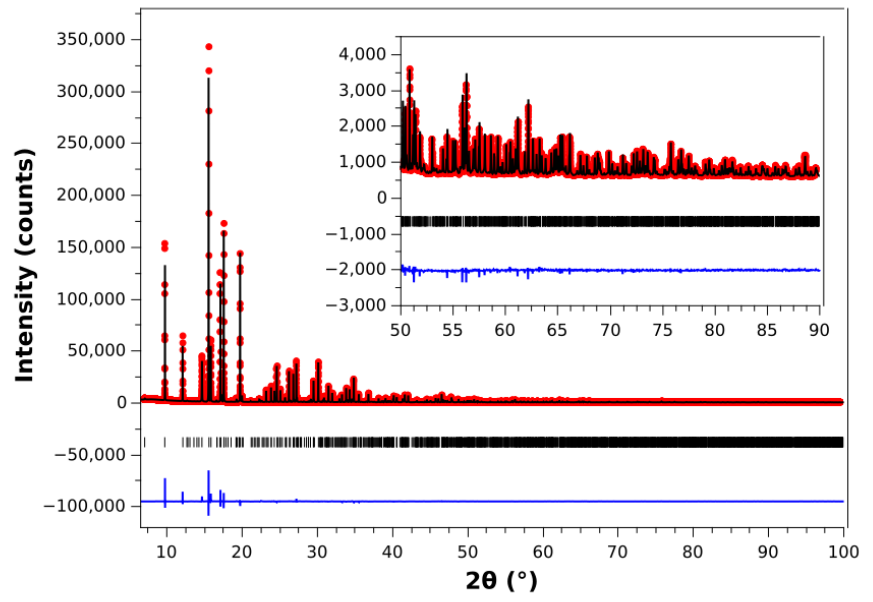

Figure SI. 3: Rietveld refined synchrotron powder XRD patterns of (a) $\mathrm{MnSi}_{1.74}-\mathrm{Oh}$, (b) $\mathrm{MnSi}_{1.74}-96 \mathrm{~h}$ and (c) $\mathrm{MnSi}_{1.74}-336 \mathrm{~h}(\lambda=0.58143 \AA$ ). The experimental data are plotted in red symbols, the calculated pattern with a black line and the difference with a blue line. The vertical ticks indicate the theoretical Bragg positions. The inset is a magnified view of the high angular region of the refined pattern. 